\title{
Polymorphism CYP2D6 gene for xenobiotic biotransformation in populations of Buryats and Russians of Eastern Siberia
}

\author{
L.E. Tabikhanova ${ }^{1 *}$, L.P. Osipova ${ }^{1,3}$, E.N. Voronina ${ }^{2,3}$, M.L. Filipenko ${ }^{2,3}$ \\ ${ }^{1}$ Institute of Cytology and Genetics SB RAS, Novosibirsk, Russia \\ ${ }^{2}$ Institute of Chemical Biology and Fundamental Medicine SB RAS, Novosibirsk, Russia \\ ${ }^{3}$ Novosibirsk State University, Novosibirsk, Russia \\ *e-mail: tabikhan@bionet.nsc.ru
}

Key words: population genomics, Asian populations, Buryats, polymorphism, genes for xenobiotic biotransformation, databases, CYP2D6

Motivation and Aim: The study of the gene polymorphism of the system of biotransformation of xenobiotics is an important area of modern medical and genetic research. The CYP2D6*3 (2549del) and CYP2D6*4 (1846A) variants are associated with risks of drugs side effects and cancer. The variants are annotated as regulatory polymorphisms $r s 35742686$ and $r s 3892097$, correspondingly. The aim of this work is to study CYP2D6 gene polymorphism in different ethnic groups.

Methods and Algorithms: This study was performed on Eastern $(\mathrm{N}=132)$ and Western $(\mathrm{N}=278)$ Buryats, Russians of East Siberia $(\mathrm{N}=122)$ and Metis, the progeny of mixed marriages of Buryats with Russians $(\mathrm{N}=56)$. Genotyping was performed using real-time PCR with competitive TaqMan allele-specific probes.

Results: The CYP2D6*3 (2549del) allele was not detected in Buryat cohorts, among Russians it was $0.4 \%$, and it was $2.7 \%$ among Métis. The frequency of the CYP2D6*4 (1846A) in Eastern and Western Buryats was $5.3 \%$ and $4.3 \%$, respectively. These data correspond to the frequency range found in Eastern Asian populations [1]. It was significantly higher in the Russian population (12\%), and among Métis (9.8\%).

Conclusion: The obtained data makes it possible to predict a reduced risk of side effects of drugs and cancer associated with CYP2D6*3 (2549del) and CYP2D6*4 (1846A) in the Buryat population. However, metisation introduces new polymorphic variants into indigenous populations, shifts gene frequencies and changes the degree of risks.

Acknowledgements: Authors are grateful to Dr Ming Chen and Haihua Bai for science discussion. Previous researches were supported by RFBR-NSFC grant project. The work is supported by budget project No. 0324-2018-0016.

\section{References}

1. The 1000 Genomes Project Consortium (2012) An integrated map of genetic variation from 1,092 human genomes. Nature. 7422:56. 